\title{
Do positrons measure atomic and molecular diameters?^
}

\author{
Jan Franz ${ }^{1}$, Kamil Fedus ${ }^{2, a}$, and Grzegorz P. Karwasz ${ }^{2}$ \\ 1 Department of Theoretical Physics and Quantum Informatics, Faculty of Applied Physics and Mathematics, Gdansk University \\ of Technology, ul. Narutowicza 11/12, 80-233 Gdańsk, Poland \\ 2 Institute of Physics, Faculty of Physics, Astronomy and Informatics, Nicolaus Copernicus University, Grudziadzka 5/7, 87-100 \\ Torun, Poland
}

Received 15 February 2016 / Received in final form 11 April 2016

Published online 20 July 2016

(C) The Author(s) 2016. This article is published with open access at Springerlink.com

\begin{abstract}
We report on density functional calculations (DFT) of elastic integral scattering cross-sections for positron collisions with argon, krypton, nitrogen and methane. The long-range asymptotic polarization potential is described using higher-order terms going much beyond an induced dipole potential $\left(-\alpha / r^{4}\right)$ while the short-range interaction is modeled by two different forms of electron - positron correlation potential (Boroński-Nieminen and Quantum Monte Carlo potentials). The results of both approaches agree quite well with the recent theoretical and measured values. Based on the present and previous theoretical and experimental data we discuss some systematics observed in integral scattering cross-sections below the positronium formation threshold. In particular we point out on the correlation between the values of scattering cross-sections and atomic dimensions.
\end{abstract}

\section{Introduction}

"An exact determination of actual atomic dimensions" is the problem that was already posed by A. Einstein in his paper on Brownian motion [1]. This happened in 1905, before formulation of Quantum Mechanics but when the first experiments with electron scattering on atoms and molecules were already available [2]. As discussed below, electron scattering failed to give clear indications on such dimensions. Numerous determinations of atomic and molecular dimensions come from kinetic theory of gases and liquids (viscosity, van der Waals, diffusion), from crystallographic studies and from quantum chemistry calculations (outermost orbitals, covalent radii). Knowing exact values of hard sphere-like dimensions is important in many issues, including normalizing cross sections in electron scattering experiments, see for example [3]. Here, we present a tentative comparison of atomic and molecular dimensions derived from experimental and theoretical total cross sections for positron scattering below the positronium formation threshold.

Probing atomic and molecular diameters by scattering of smaller particles, like electrons, was first considered by Lenard [2]. In fact, for scattering on targets like $\mathrm{H}_{2}$,

\footnotetext{
* Contribution to the Topical Issue "Advances in Positron and Electron Scattering", edited by Paulo Limao-Vieira, Gustavo Garcia, E. Krishnakumar, James Sullivan, Hajime Tanuma and Zoran Petrovic.

${ }^{a}$ e-mail: kamil@fizyka.umk.pl
}

$\mathrm{N}_{2}, \mathrm{SO}_{2}, \mathrm{CO}_{2}$ at high collision energies $(100-3000 \mathrm{eV})$ he observed some scaling of cross sections with gas densities. Surprisingly, low-energy (below $1 \mathrm{eV}$ ) experiments by Ramsauer [4] showed deep minima in cross sections for Ar, $\mathrm{Kr}, \mathrm{Xe}$ and $\mathrm{CH}_{4}$. This triggered the quantum wave mechanics, but hindered trials to derive atomic dimensions via electron scattering.

Ramsauer minima appear when the scattering potential is enough strong to induce a $2 \pi$ (or its multiples) phase shift in the $s$-wave scattering phase [5]. For electron scattering both the static and polarization interactions are attractive and they cancel out with the repulsive exchange interaction at the Ramsauer minimum. Search of similar minima in total cross sections was one of motivations of first experiments on low-energy positron scattering, but results were not conclusive at that time [6].

First experiments on total cross sections for positron scattering [6-8] used strong guiding magnetic fields and rather wide apertures in the scattering cell; further, the yield of positron sources (and moderators) was low, what made difficult measurements at low energies. These factors potentially led to underestimation of total cross sections, as seen in comparative measurements done, for example, by Sueoka and Mori [9-11] (and discussed a posteriori in [12]). The fall of total cross sections towards zeroenergy, reported for many targets [11] was in contradiction with theories. Also for targets such as $\mathrm{H}_{2}$ and $\mathrm{N}_{2}$ the early experiments [6-8] disagreed with theories, suggesting existence of Ramsauer minima, see for example [13] for comparison. 
More recent positron experiments [13-15] used stronger positron sources (with high-efficiency moderators) and narrow apertures in the scattering cell (for example $1.5 \mathrm{~mm}$ diameter with $10 \mathrm{~cm}$ scattering cell length in [13]). Further, a detailed analysis of the influence of the magnetic field on the angular resolution was developed; allowing making appropriate corrections in the low-energy region, see for example [14]. This allows to evidence three regions in the low energy positron total cross sections for atomic and some non-polar molecular targets: a very low energy (below $1 \mathrm{eV}$ ) range where the TCS rises in the zeroenergy limit, a rather flat dependence up to the positronium threshold, and at higher energies a rise due to the positronium formation [16,17]. A question arises, if these features can be reproduced by a unique theory and if the cross sections in the "flat" region scale with atomic and molecular dimensions [18].

From the theoretical point of view, in spite of the absence of exchange effects, positron scattering is quite a delicate task. The static potential due to the interactions with (partially unscreened) atomic nuclei is repulsive for positrons so it tends to cancel out with the attractive polarization interaction. As a consequence, at short distances, an attractive potential due to electron-positron correlation effects becomes important. Modeling this interaction (together with the virtual-positronium formation) is a long-standing problem (see for example [19]). For example, citing some most recent works on the atoms: McEachran et al. (see [20] and references therein) used different forms of polarized-orbital calculations. Gribakin and co-workers [21] used many-body theory that model also formation of virtual positronium and multi-electron correlation. Bray and Fursa [22], for the Ne-Xe series, considered only one-electron excitations out of six $p$-electrons above an inert Hartree-Fock core; in their approach formation of virtual positronium is taken into account via excitation to continuum-like pseudostates.

At present we calculate elastic cross sections for positron scattering on $\mathrm{Ar}, \mathrm{Kr}, \mathrm{N}_{2}$ and $\mathrm{CH}_{4}$. We use a multi-term expansion of the long range potential and two different forms of short-range electron-positron correlation potentials within the frame of density functional theory (DFT). Derived cross-sections are compared to available experiments; uncertainties in the experiments are discussed. Within these uncertainties the present theories agree well with recent experiments in all gases except $\mathrm{CH}_{4}$. Based on the present theory and available experimental results we search for a correlation between positron scattering data and atomic dimensions.

\section{Theoretical and computational methods}

\subsection{Density functional theory for the positron-molecule interactions}

We are solving the Schrödinger equation

$$
H \Psi=E_{\text {total }} \Psi
$$

in the body-fixed reference frame of the target. Here $E_{\text {total }}$ and $\Psi$ are the total energy and the total wavefunction, respectively. In the following we are working within the Born-Oppenheimer approximation.

The interaction between the positron and the electrons are described by electron-positron density functional theory. We assume that we can re-write the Hamiltonian as

$$
H=H_{\text {target }}+H_{p}
$$

where the target Hamiltonian is defined as

$$
H_{\text {target }}=T_{e}+V_{e e}+V_{e n}+V_{n n}
$$

and $H_{p}$ describes all terms involving the positron

$$
H_{p}=T_{p}+V_{\text {eff }}
$$

The effective potential $V_{\text {eff }}$ depends only on the electrondensity, the nuclear coordinates, nuclear charges and the positron position. It can be written as

$$
V_{\mathrm{eff}}=V_{e p}+V_{p n}+V_{\mathrm{pcp}} .
$$

The extra term $V_{p c p}$ is the electron-positron correlationpolarization potential and describes the many particle effects between the positron and the electrons. In the twocomponent density functional theory [23] it plays a similar role as the exchange-correlation potential in Kohn-Sham theory [24].

\subsection{Correlation-polarization potential}

The correlation-polarization potential is devided in two parts, depending on the radial distance $r_{\mathrm{p}}$. For large radial distances, the correlation-polarization potential is dominated by the polarization potential

$$
\text { for } r_{\mathrm{p}} \rightarrow \infty: V_{\mathrm{pcp}}\left(\mathbf{r}_{\mathrm{p}}\right) \rightarrow V_{\mathrm{pol}}\left(\mathbf{r}_{\mathrm{p}}\right) .
$$

For short radial distances, the correlation-polarization potential is dominated by the correlation potential

$$
\text { for } r_{\mathrm{p}} \rightarrow 0 \quad: \quad V_{\mathrm{pcp}}\left(\mathbf{r}_{\mathrm{p}}\right) \rightarrow V_{\text {corr }}\left(\mathbf{r}_{\mathrm{p}}\right) .
$$

The two parts are connected at the radial distance $r_{c}$, which is the outermost point, at which $V_{\text {pol }}$ becomes larger than $V_{\text {corr }}$. For the computation for the collisions with atoms (here $\mathrm{Ar}$ and $\mathrm{Kr}$ ) we are using for the polarization potential the asympotic form [25]

$$
V_{\mathrm{pol}}\left(\mathbf{r}_{\mathrm{p}}\right)=-\frac{\alpha_{\text {Dip }, 0}}{2 r_{\mathrm{p}}^{4}}-\frac{\alpha_{\text {Quad }}}{2 r_{\mathrm{p}}^{6}}-\frac{\alpha_{\text {Oct }}}{2 r_{\mathrm{p}}^{8}}+\frac{B}{2 r_{\mathrm{p}}^{7}}-\frac{\gamma}{24 r_{\mathrm{p}}^{8}} .
$$

Here $\alpha_{\text {Dip }, 0}$ is the isotropic static dipole polarizability, $\alpha_{\text {Quad }}$ is the isotropic static quadrupole polarizability, and $\alpha_{\text {Oct }}$ is the isotropic static octupole polarizability. $B$ is the dipole-dipole - quadrupole hyperpolarizability and $\gamma$ is the dipole-dipole - dipole-dipole hyperpolarizability. The importance of including higher-order terms beyond the asymptotic polarization potential described by the $-\alpha / r^{4}$ 
behaviour is pointed out by Gianturco et al. [25]. Morison and Hay [26] have also demonstrated the importance of additional terms in the asymptotic polarization potential for electron and positron scattering from molecular targets such as $\mathrm{N}_{2}$. Hence, for the computation for the collisions with molecules (here $\mathrm{N}_{2}$ and $\mathrm{CH}_{4}$ ) the polarization potential we are using the form

$$
\begin{aligned}
V_{\mathrm{pol}}\left(\mathbf{r}_{\mathrm{p}}\right)= & -\frac{\alpha_{\text {Dip }, 0}}{2 r_{\mathrm{p}}^{4}}-\frac{\alpha_{\text {Dip }, 2}}{2 r_{\mathrm{p}}^{4}} P_{2}\left(\cos \theta_{\mathrm{p}}\right)-\frac{\alpha_{\mathrm{Quad}}}{2 r_{\mathrm{p}}^{6}}+\frac{B}{2 r_{\mathrm{p}}^{7}} \\
& -\frac{\gamma}{24 r_{\mathrm{p}}^{8}} .
\end{aligned}
$$

Here $\alpha_{\mathrm{Dip}, 2}$ is the anisotropic static dipole polarizability and $P_{2}\left(\cos \theta_{\mathrm{p}}\right)$ is a Legendre polynomial. For the correlation potential we are presenting calculations done with two different potentials. The first potential is derived from the density functional of Boroński and Nieminen [27] in the form presented by Jain and Gianturco [28]. The second potential is derived from recent Quantum Monte Carlo (QMC) by Drummond et al. [29]. Drummond et al. [29] are presenting a simple analytic expression for the relaxation energy of a positron in homogenous electron gas. The potential used here is the functional derivative of the corresponding energy functional (for details see Franz [30]).

\subsection{Scattering cross sections}

We are using a partial wave expansion for the wave function employing symmetry adapted linear combinations of real spherical harmonics (see Gianturco and Jain [31] for details). The radial Schrödinger equation is solved by numerical integration of the coupled Volterra integral equations (see e.g. Chap. 5 in Gianturco [32] or Franz [33] or Fedus et al. [34])

$$
\begin{aligned}
\mathbf{U}(R)= & \int_{R_{0}}^{R}\{\mathbf{J}(R) \cdot \mathbf{N}(x) \\
& +\mathbf{N}(R) \cdot \mathbf{J}(x)\} \cdot \mathbf{V}(x) \cdot \mathbf{U}(x) d x .
\end{aligned}
$$

Here $\mathbf{J}$ and $\mathbf{N}$ are diagonal matrices containing the spherical Ricatti-Bessel and Ricatti-Neumann functions, respectively, $\mathbf{V}$ is the potential matrix and the matrix $\mathbf{U}(R)$ is related to the radial wave function. In the asymptotic region the $\mathbf{K}$-matrix is computed, from which we obtain the T-matrix as:

$$
\mathbf{T}=1-(1-i \mathbf{K}) \cdot(1+i \mathbf{K})^{-1} .
$$

The elastic integral cross section is given as:

$$
\sigma^{\text {elastic }}=\frac{\pi}{k^{2}} \sum_{l v} \sum_{l^{\prime} v^{\prime}}\left|T_{l^{\prime} v^{\prime}}^{l v}\right|^{2} .
$$

\subsection{Computational details}

\subsubsection{Electronic structure calculations for atoms}

The electron densities for the atoms (Ar and $\mathrm{Kr}$ ) are computed with the MOLCAS software package [35]. Electronelectron correlation is considered through the complete
Table 1. Parameters for the asymptotic potential. All values are in atomic units.

\begin{tabular}{ccccccc}
\hline Target & $\alpha_{\text {Dip }, 0}$ & $\alpha_{\text {Dip }, 2}$ & $\alpha_{\text {Quad }}$ & $\alpha_{\text {Oct }}$ & $B$ & $\gamma$ \\
\hline $\mathrm{Ar}$ & $11.070^{\mathrm{a}}$ & - & $52.4^{\mathrm{b}}$ & $490^{\mathrm{b}}$ & $-164.3^{\mathrm{b}}$ & $1083^{\mathrm{b}}$ \\
$\mathrm{Kr}$ & $17.075^{\mathrm{a}}$ & - & $92.7^{\mathrm{b}}$ & $793^{\mathrm{b}}$ & $-343.2^{\mathrm{b}}$ & $2255^{\mathrm{b}}$ \\
$\mathrm{N}_{2}$ & $11.74^{\mathrm{d}}$ & $4.593^{\mathrm{d}}$ & $83.26^{\mathrm{e}}$ & - & $-151.53^{\mathrm{g}}$ & $927^{\mathrm{e}}$ \\
$\mathrm{CH}_{4}$ & $16.39^{\mathrm{f}}$ & - & $120.90^{\mathrm{f}}$ & - & $-317.79^{\mathrm{g}}$ & $2312^{\mathrm{f}}$ \\
\hline
\end{tabular}

${ }^{\text {a }}$ Experimental values from Holm and Kerl [50,51] as cited in Schwerdtfeger [52].

${ }^{\mathrm{b}}$ From tabulated values in Gianturco et al. [25].

c From explicitly correlated calculations by Bishop et al. [53-55].

d Obtained from dipole oscillator strength distribution by Kumar and Meath [56].

e From $\operatorname{CCSD}(\mathrm{T})$ calculations by Maroulis [57].

${ }^{\mathrm{f}}$ From $\operatorname{CCSD}(\mathrm{T})$ calculations by Maroulis [58].

g From CCSD calculations by Coriani et al. [59].

active space self-consistent-field (CASSCF) method. In the calculations for Ar the active space is generated by 8 active electrons in the orbitals $(3 s, 3 p, 3 d, 4 s, 4 p, 5 s, 5 p)$, which results in 64331 determinants. For Kr the active space is generated by 8 active electrons in the orbitals $(4 s, 4 p, 4 d, 5 s, 5 p, 6 s, 6 p)$, which results in 415306 determinants. For technical reasons the calculations are done in $D_{2 \mathrm{~h}}$ point group symmetry.

The contracted gaussian basis set for Douglas-KrollHeß calculations of triple zeta valence quality plus polarization functions (TZP-DKH) of Jorge et al. [36] are used. The TZP-DKH basis set has the contraction scheme $(13 s, 10 p, 2 d, 1 f) \rightarrow(8 s, 5 p, 2 d, 1 f)$ for $\mathrm{Ar}$ and $(16 s, 13 p, 6 d, 2 f, 1 g) \rightarrow(9 s, 6 p, 3 d, 2 f, 1 g)$ for Kr. Both basis sets are obtained from the EMSL Basis Set Library $[37,38]$. Scalar relativistic effects of the target wave function are included through the Douglas-Kroll-Heß method $[39,40]$.

The electron density of the target is described by the reduced one-particle density matrix (see e.g. Chaps. 5 and 8 in McWeeny [41]) of the CASSCF wave function for the electronic ground state (the ${ }^{1} \mathrm{~S}_{0}$ state). Natural orbitals are used to represent the reduced one-particle density matrix (see e.g. Chap. 3 in Davidson [42]). For this purpose we have implemented an interface between our scattering codes and the MOLCAS software package. Furthermore we have extended our scattering program to enable the usage of $g$-type functions.

\subsubsection{Electronic structure calculations for molecules}

All electronic structure calculations for the target molecules are done with the TURBOMOLE software package [43]. For $\mathrm{N}_{2}$ the calculations are done for a bond length of $1.094 \AA$. The molecular orbitals, which were used in the scattering calculations to represent the target electron density, are generated with the B3LYP (Becke [44], three-parameter, Lee-Yang-Parr [45]) exchange-correlation functional, using the double-zeta basis set of Dunning and Hay [46] with polarization and diffuse functions. 


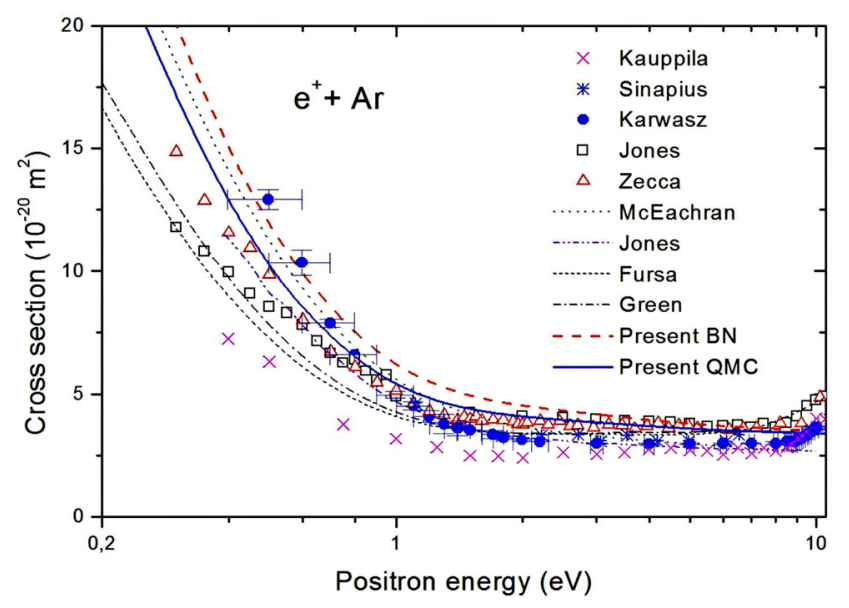

Fig. 1. Elastic scattering cross section for Ar. Present DFT BN and DFT QMC calculations are compared with chosen theoretical and experimental results. Theories: McEachran et al. [62] (polarized orbital), Jones et al. [61] (relativistic optical potential), Fursa and Bray [22] (convergent close-coupling) and Green et al. [21] (many-body theory). Experiments: Kauppila et al. [6], Sinapius et al. [63], Karwasz et al. [13], Jones et al. [61] and Zecca et al. [60].

For $\mathrm{CH}_{4}$ the molecular geometry (CH-bondlength is $R=1.089 \AA$ ) is optimized using the B3LYP functional and the aug-cc-pVTZ basis set [47]. The molecular orbitals, which were used in the scattering calculations to represent the target electron density, are generated with the B3LYP functional and the TZP basis set of Barbieri et al. [48], which we augmented by diffuse functions of Dunning and Hay [46].

\subsubsection{Quantum scattering calculations}

The single-centre-expansions (SCE) of the molecular electron density and of the potential are computed with the VOLPOS software package [49], which is also used to solve the coupled scattering equations by Volterra integration. The grid for the radial integration ranges up to distance of $146 \mathrm{bohr}$. The angular basis set includes terms up to $L_{\max }=24$ for the expansion of the potential and up to $l_{\max }=12$ for the expansion of the wavefunction. At the outermost radial distance the $\mathbf{K}$-matrices in the bodyfixed frame are generated and elastic integral cross section $\sigma^{\text {elastic }}$ are computed.

\section{Results}

Present theories (with BN - Boroński-Nieminen and QMC - Quantum Monte Carlo functionals) for Ar (see Fig. 1) reproduces well the very low-energy fall of the cross section from measurements by Karwasz et al. [13] and agrees well (within error bars) with "constant" cross section values by Zecca et al. [60] and Jones et al. [61] at higher energies. Note, that the data by Karwasz et al. slightly differs from those by Zecca et al., who used the same settings of the apparatus (and $-0.2 \mathrm{eV}$ energy shift), but applied a $+3 \%$

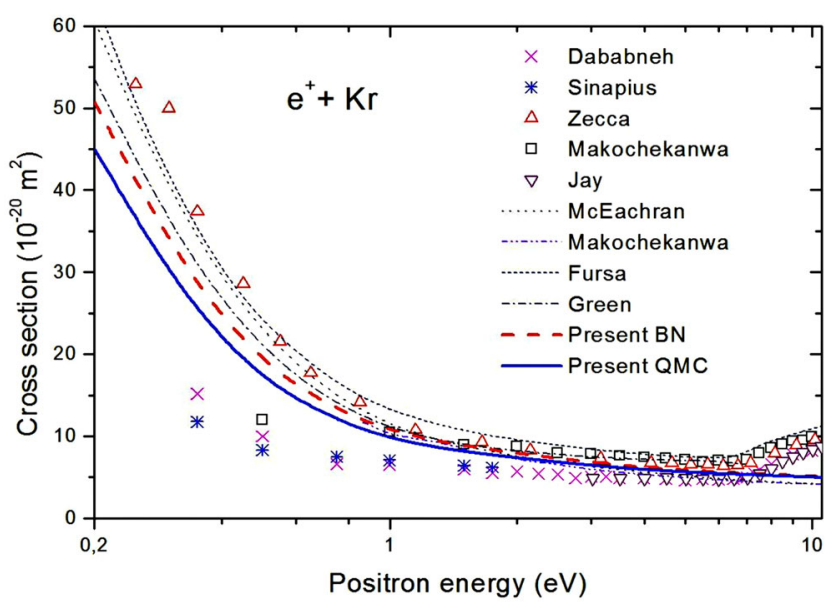

Fig. 2. Elastic scattering cross section for Kr. Present DFT BN and DFT QMC calculations are compared with chosen theoretical and experimental results. Theories: McEachran et al. [68] (polarized orbitals), Makochekanwa et al. [65] (relativistic optical potential), Fursa and Bray [22] (convergent closecoupling) and Green et al. [21] (many-body theory). Experiments: Dababneh et al. [66], Sinapius et al. [63], Makochekanwa et al. [65], Zecca et al. [64] and Jay and Coleman [67].

thermal transpiration correction. Within these systematic uncertainties, the agreement with the three recent experiments is really good.

Also for $\mathrm{Kr}$ (see Fig. 2) the agreement with recent experiments (Zecca et al. [64], Makochekanwa et al. [65]) is quite good, taking into account some $( \pm 0.2 \mathrm{eV})$ uncertainty in the energy determination in the Trento apparatus and indirect method of the angular resolution correction in ANU measurements, which can effect particularly the low-energy cross sections.

For $\mathrm{N}_{2}$ (see Fig. 3) the two theoretical approaches are giving quite different results. At collision energies below $0.5 \mathrm{eV}$ the cross sections computed with the BN-functional are about 50 percent higher than those obtained with the QMC-functional. The origin of these differences can be traced back to the different parameterizations used for the relaxation energy of a positron in the homogeneous electron gas at various electron densities. For low electron densities the computed relaxation energy between the two functionals can differ by up to 0.04 Hartree (approximately $1 \mathrm{eV}$ ) (see Drummond et al. [29]). The computed cross sections obtained with the BN-functional fall in-between the energy-scale uncertainty of the two Trento determinations (Karwasz et al. [13] and Zecca et al. [15]), agreeing better with the latter at higher energies. The calculations with the QMC-functional follow more closely the determinations by Zecca et al. [15] over the complete energy range.

In $\mathrm{CH}_{4}$ (see Fig. 4) different experimental data strongly disagree. Note that Zecca's results [74] were obtained on a modified Trento apparatus when compared to earlier experiments in Ar and $\mathrm{N}_{2}$ by Karwasz et al. [13]. This modified system was composed of a shorter scattering cell $(2.21 \mathrm{~cm}$ as compared to earlier $10 \mathrm{~cm})$ and a weaker positron source. Short scattering cells can lead 
Table 2. Selectively chosen atomic and molecular radii determined using different methods and definitions. All values are in Bohr units $\left(a_{0}\right)$.

\begin{tabular}{ccccccc}
\hline Target & Experiment & SCF $^{\mathrm{c}}$ & Van der Waals $^{\mathrm{d}}$ & Covalent radii & Positron scattering $^{\mathrm{g}}$ & Matching point BN/QMC $^{\mathrm{h}}$ \\
\hline $\mathrm{Ar}$ & $3.44^{\mathrm{a}} / 3.28^{\mathrm{b}}$ & 1.34 & 3.55 & $2.00^{\mathrm{e}} / 1.81^{\mathrm{f}}$ & $2.04^{[61]} / 1.85^{[13]} / 2.08^{[60]}$ & $3.56 / 3.63$ \\
$\mathrm{Kr}$ & - & 1.66 & 3.82 & $2.19^{\mathrm{e}} / 2.21^{\mathrm{f}}$ & $2.84^{[65]} / 2.70^{[64]} / 2.32^{[67]}$ & $3.92 / 4.01$ \\
$\mathrm{~N}_{2}$ & $3.54^{\mathrm{a}} / 3.29^{\mathrm{b}}$ & - & - & - & $2.12^{[13]} / 2.28^{[15]} / 2.15^{[73]}$ & $3.69 / 3.69$ \\
$\mathrm{CH}_{4}$ & $3.91^{\mathrm{a}} / 3.58^{\mathrm{b}}$ & - & - & - & $2.98^{[74]} / 2.48^{[76]} / 2.21^{[78]}$ & $3.82 / 3.92$ \\
\hline
\end{tabular}

${ }^{a}$ Rigid-sphere molecular diameters calculated from viscosity measurements [82].

b Rigid-sphere molecular diameters calculated from self-diffusion measurements [82].

c Self-consistent-field theory by Clementi et al. [83].

${ }^{\mathrm{d}}$ Van der Waals radii by Bondi [84] derived from X-ray diffraction data.

e Covalent radii by Cordero et al. [85] derived from crystallographic data.

${ }^{\mathrm{f}}$ Single bond covalent radii by Pyykkö and Atsumi [86].

g Rigid-sphere radii determined from positron scattering cross-sections measured at positronium formation threshold.

${ }^{\mathrm{h}}$ Matching point of long-range asymptotic potential and short-range correlation potentials (BN/QMC) in present calculations.

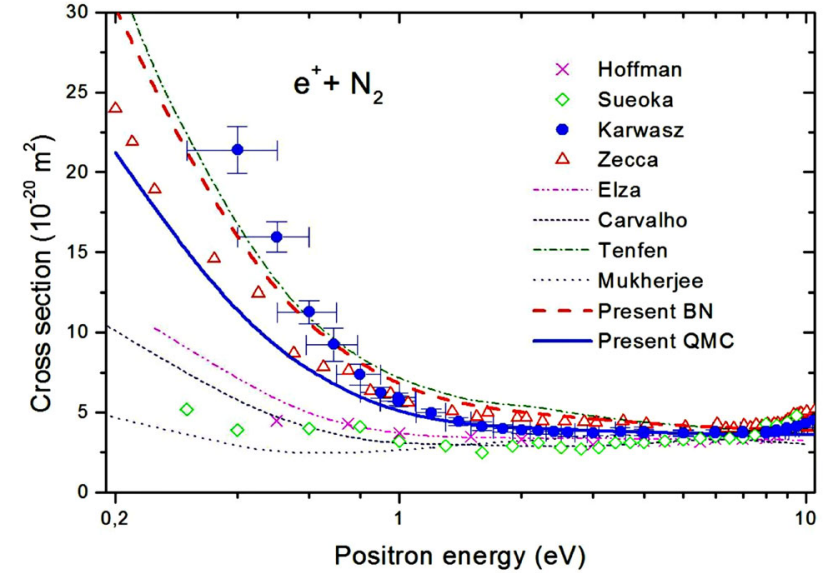

Fig. 3. Elastic scattering cross section for $\mathrm{N}_{2}$. Present DFT BN and DFT QMC calculations are compared with chosen theoretical and experimental results. Theories: Elza et al. [69] (nonadiabatic correlation effects), Carvalho et al. [70] (Schwinger multichannel), Tenfen et al. [71] (ab initio target polarization) and Mukherjee et al. [72] (positron correlation-polarization potential). Experiments: Hoffman et al. [73], Sueoka and Hamada [8], Karwasz et al. [13] and Zecca et al. [15].

to systematic errors due to gas-outflow effects, that depend on particular working conditions (pumping speed, type of gas, pressures used), see for example [75]. Experiments with weak positron sources usually require the use of strong magnetic fields, what increases the effective path length of projectiles in the gas cell, compare reference $[76-78]$, so measured cross sections can be overestimated. On the other hand, wider apertures in scattering cell worsen the angular resolution, what underestimates cross sections in an energy-dependent manner. Confluence of these different effects results in the discrepancy of crosssections measured using different experimental systems (Fig. 4).

In Table 2 we compare different determinations of atomic and molecular "radii" - experimental from viscosity, X-ray diffraction and self-diffusion measurements together with theoretical from self-consistent field calculations (for atoms) and present - from positron to-

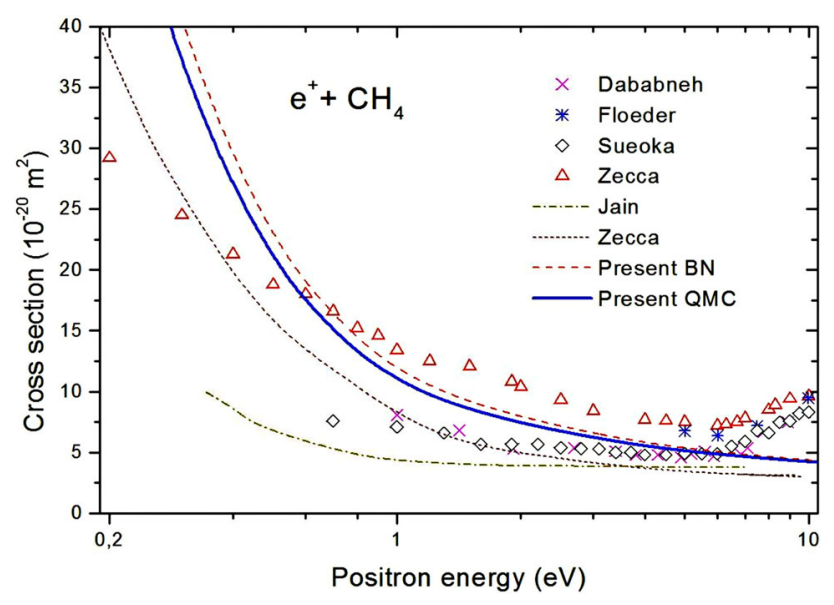

Fig. 4. Elastic scattering cross section for $\mathrm{CH}_{4}$. Present DFT BN and DFT QMC calculations are compared with chosen theoretical and experimental results. Theories: Gianturco et al. [79] (parameter-free model potential) and Zecca et al. [74] (Schwinger multichannel). Experiments: Dababneh et al. [76], Floeder et al. [77], Sueoka and Mori [78] and Zecca et al. [74].

tal cross sections. The latter values, $R$, were evaluated at the threshold of positronium formation from a simple $\sigma^{\text {elastic }}=\pi R^{2}$ relation (considering the rigid-sphere model though the picture of sticky ball would be more appropriate). In the last column we quote also a matching point between the long-range potential and the short-range correlation potential in present DFT calculations. Interestingly, for atoms the latter values are close to the van der Waals radii while radii derived from scattering data are comparable with covalent radii. This can be roughly understood noting that van der Waals radius defines the spherical volume occupied by atom that is impenetrable for other atoms. Therefore, it should more or less correspond to the shortest distance from the nucleus where the atom can be treated as the single object from the point of view of an incoming light particle such as a positron. At this distance the short-range part of particle-atom interaction potential becomes negligible and only the longrange part remains. On the other hand the covalent radius refers to half of the length between two singly bonded 
atoms of the same element. Thus it defines the distance from the nucleus where valence electrons form a covalent bond. It is expected that at this distance the incoming positron strongly interacts with the valence electrons, for example through the virtual Positronium formation [21], what determines the output of scattering experiment at impact energies just below the threshold for positronium formation.

It is clear from Table 2 that the positron cross-sectional data (at the flat part of the integral cross section below the positronium threshold) scale in a similar way as atomic and molecular dimensions regardless of the way they were estimated.

\section{Conclusions}

The recent experimental data show that positron interaction with atoms and molecules characterized by moderate polarizability such as $\mathrm{H}_{2}, \mathrm{Ar}, \mathrm{Kr}, \mathrm{CH}_{4}$ can be divided into two energy regions up to the threshold for positronium formation. In the very-low energy region the rise of cross section is well explained by long-range potentials, with the dipole polarization dominating [34,80]. At higher energies - just below the threshold for positronium formation, the strong correlation potential hinders the fall of the cross section, making it almost constant vs. energy. In the present paper we show that by modeling the shortrange interaction with two different approaches (BorońskiNieminen and Quantum Monte Carlo potentials) it is possible to reconstruct shape and magnitude of experimental cross-sections within the frame of DFT theory if the longrange asymptotic polarization potential is described by higher-order terms going much beyond an induced dipole potential $\left(-\alpha / r^{4}\right)$. The necessity to include these additional terms confirms the presence of strong electronpositron correlation for the interaction energy just below the positronium formation threshold where the plateauing of cross-sections occurs. We notice that atomic radii for Ar and $\mathrm{Kr}$ estimated from this plateau whitin the frame of a simple rigid-sphere model are comparable to the covalent radii of these elements.

Experimental data for low-polarizability systems such as $\mathrm{He}$ [81] and Ne [61], suggest an absence of strong shortrange correlation effects since no flattening of the crosssections is observed. The latter effect is also not so pronounced for highly polarizable systems such as Xe [87]. However, the theoretical calculations by McEachran et al. [68] and Schrader [88] show that pure elastic scattering cross-section of Xe flatten out above the Ps threshold, plateauing at magnitudes that are comparable with the covalent radius of $\mathrm{Xe}, 2.65 a_{0}$ [85], when using the hard sphere picture.

To summarize, correlation between an incident positron and target's electrons is a short-distance interaction, so physically it is to be imagined occurring at distances comparable to the outer electronic shells of the target. Answering the title question, we can say Yes! Positrons measure molecular diameters via "sticking" to outermost electrons of the target, i.e. due to multi-body correlation effects.
This work is supported by the grant 2014/15/D/ST2/02358 of National Science Center in Poland. J.F. thanks Prof. Sigrid Peyerimhoff (University of Bonn) for computational resources and Prof. Edward Boroński (Opole University) for helpful discussions.

\section{References}

1. A. Einstein, Ann. Phys. 322, 549 (1905)

2. P. Lenard, Ann. Phys. 292, 255 (1895)

3. C.J. Colyer, V. Vizcaino, J.P. Sullivan, M.J. Brunger, S.J. Buckman, New J. Phys. 9, 41 (2007)

4. C. Ramsauer, Ann. Phys. 66, 546 (1921)

5. J. Holtsmark, Rev. Mod. Phys. 4, 577 (1932)

6. W.E. Kauppila, T.S. Stein, G. Jesion, Phys. Rev. Lett. 36, $580(1976)$

7. M. Charlton, T.C. Griffith, G.R. Heyland, G.L. Wright, J. Phys. B 16, 323 (1983)

8. O. Sueoka, A. Hamada, J. Phys. Soc. Jpn 62, 2669 (1993)

9. O. Sueoka, J. Phys. B 21, L631 (1988)

10. O. Sueoka, M.K. Kawada, M. Kimura, Nucl. Instrum. Methods Phys. B 171, 96 (2000)

11. C. Makochekanwa, O. Sueoka, M. Kimura, Phys. Rev. A 68, 32707 (2003)

12. G.P. Karwasz, A. Karbowski, Z. Idziaszek, R.S. Brusa, Nucl. Instrum. Methods B 266, 471 (2008)

13. G.P. Karwasz, D. Pliszka, R.S. Brusa, Nucl. Instrum. Methods B 247, 68 (2006)

14. J.P. Sullivan, A. Jones, P. Caradonna, C. Makochekanwa, S.J. Buckman, Rev. Sci. Instrum. 79, 113105 (2008)

15. A. Zecca, L. Chiari, A. Sarkar, M.J. Brunger New J. Phys. 13, $115001(2011)$

16. G.P. Karwasz, Eur. Phys. J. D 35, 267 (2005)

17. G.P. Karwasz, Eur. Phys. J. D 27, 53 (2006)

18. G.P. Karwasz, in 13th General Conference of the European Physical Society, Bern Switzerland, 11-15 July 2005, BB10-TUE, Europhysics Conference Abstracts Volume 29D, p. 106

19. N.F. Mott, H.S.W. Massey, in Theory of Atomic Collisions (London, Oxford University Press, 1965), pp. 550-555

20. R.P. McEachran, A.D. Stauffer, J. Phys. B 42, 075202 (2009)

21. D.G. Green, J.A. Ludlow, G.F. Gribakin, Phys. Rev. A 90, $032712(2014)$

22. D.V. Fursa, I. Bray, New J. Phys. 14, 035002 (2012)

23. R.M. Nieminen, E. Boroński, L.J. Lantto, Phys. Rev. B 32, 1377 (1985)

24. W. Kohn, L.J. Sham, Phys. Rev. 140, A1133 (1965)

25. F.A. Gianturco, A. Jain, J.A. Rodriguez-Ruiz, Phys. Rev. A 84, 4321 (1993)

26. M.A. Morrison, P.J. Hay, Phys. Rev. A 20, 740 (1979)

27. E. Boroński, R.M. Nieminen, Phys. Rev. 34, 3820 (1986)

28. A. Jain, F.A. Gianturco, J. Phys. B 24, 2387 (1991)

29. N.D. Drummond, P. López Ríos, C.J. Pickard, R.J. Needs, Phys. Rev. Lett. 107, 207402 (2011)

30. J. Franz, manuscript in preparation

31. F.A. Gianturco, A. Jain, Phys. Rep. 143, 347 (1986)

32. F.A. Gianturco, The Transfer of Molecular Energies by Collision: Recent Quantum Treatments (Springer-Verlag, Berlin, 1979)

33. J. Franz, J. Math. Phys. 56, 012104 (2015) 
34. K. Fedus, J. Franz, G.P. Karwasz, Phys. Rev. A 91, 062701 (2015)

35. K. Andersson, M. Barysz, A. Bernhardsson, M.R.A. Blomberg, Y. Carissan, D.L. Cooper, M.P. Fülscher, L. Gagliardi, C. de Graaf, B.A. Heß, G. Karlström, R. Lindh, P.-Å. Malmqvist, T. Nakajima, P. Neogrády, J. Olsen, B.O. Roos, B. Schimmelpfennig, M. Schütz, L. Seijo, L. SerranoAndrés, P.E.M. Siegbahn, J. Stålring, T. Thorsteinsson, V. Veryazov, P.-O. Widmark, MOLCAS Version 5.4 (Lund University, Sweden, 2002)

36. F.E. Jorge, A. Canal Neto, G.G. Camiletti, S.F. Machado, J. Chem. Phys. 130, 064108 (2009)

37. D. Feller, J. Comp. Chem. 17, 1571 (1996)

38. K.L. Schuchardt, B.T. Didier, T. Elsethagen, L. Sun, V. Gurumoorthi, J. Chase, J. Li, T.L. Windus, J. Chem. Inf. Model. 47, 1045 (2007)

39. M. Douglas, N.M. Kroll, Ann. Phys. NY, 82, 89, 1974

40. G. Jansen, B.A. Heß, Phys. Rev. A 93, 6016, 1993

41. R. McWeeny, Methods of Molecular Quantum Mechanics (Academic Press, London, 1996)

42. E.R. Davidson, Reduced Density Matrices in Quantum Chemistry (Academic Press, New York, 1976)

43. Turbomole version 5-6, Copyright (C) 2002 University of Karlsruhe

44. A.D. Becke, J. Chem. Phys. 98, 5648 (1993)

45. C. Lee, W. Yang, R.G. Parr, Phys. Rev. B 37, 785 (1988)

46. T.H. Dunning Jr., P.J. Hay, in Modern Theoretical Chemistry, edited by H.F. Schaefer III (Plenum Press, New York, 1976), Vol. 3, pp. 1-28

47. T.H. Dunning Jr., J. Chem. Phys. 90, 1007 (1989)

48. P.L. Barbieri, P.A. Fantin, F.E. Jorge, Mol. Phys. 104, $2945(2006)$

49. R. Curik, F.A. Gianturco, N. Sanna, J. Phys. B: At. Mol. Opt. Phys. 33, 2705 (2000)

50. U. Hohm, K. Kerl, Mol. Phys. 69, 803 (1990)

51. U. Hohm, K. Kerl, Mol. Phys. 69, 819 (1990)

52. P. Schwerdtfeger, Atomic Static Dipole Polarizabilities, in Computational Aspects of Electric Polarizability Calculations: Atoms, Molecules and Clusters, edited by G. Maroulis (IOS Press, Amsterdam, 2006), pp. 1-32, with the addition: Updated static dipole polarizabilities are available as pdf file from the CTCP website at Massey University: http://ctcp.massey.ac.nz/ dipole-polarizabilities

53. D.M. Bishop, J. Pipin, S.M. Cybulski, Phys. Rev. A 43, 4845 (1991)

54. D.M. Bishop, S. Lam, J. Chem. Phys. 89, 1571 (1988)

55. D.M. Bishop, J. Pipin, Int. J. Quantum Chem. 45, 349 (1993)

56. A. Kumar, W.J. Meath, Theor. Chim. Acta 82, 131 (1992)

57. G. Maroulis, J. Chem. Phys. 118, 2673 (2003)

58. G. Maroulis, Chem. Phys. Lett. 226, 420 (1994)

59. S. Coriani, C. Hättig, P. Jorgensen, A. Rizzo, K. Ruud, J. Chem. Phys. 109, 7176 (1998)

60. A. Zecca, L. Chiari, E. Trainotti, D.V. Fursa, I. Bray, A. Sarkar, S. Chattopadhyay, K. Ratnavelu, M.J. Brunger, J. Phys. B 45, 015203 (2012)

61. A.C.L. Jones, C. Makochekanwa, P. Caradonna, D.S Slaughter, J.R. Machachek, R.P. McEachran, J.P. Sullivan, S.J. Buckman, A.D. Stauffer, I. Bray, D.V. Fursa, Phys. Rev. A 83, 032701 (2011)

62. R.P. McEachran, A.G. Ryman, A.D. Stauffer, J. Phys. B 12, 1031 (1979)
63. G. Sinapius, W. Raith, W.G. Wilson, J. Phys. B 13, 4079 (1980)

64. A. Zecca, L. Chiari, E. Trainotti, D.V. Fursa, I. Bray, M.J. Brunger, Eur. Phys. J. D 64, 317 (2011)

65. C. Makochekanwa, J.R. Machacek, A.C.L. Jones, P. Caradonna, D.S. Slaughter, R.P. McEachran, J.P. Sullivan, S.J. Buckman, S. Bellm, B. Lohmann, D.V. Fursa, I. Bray, D.W. Mueller, A.D. Stauffer, M. Hoshino, Phys. Rev. A 83, 032721 (2011)

66. M.S. Dababneh, W.E. Kauppila, J.P. Downing, F. Laperriere, V. Pol, J.H. Smart, T.S. Stein, Phys. Rev. A 22, 1872 (1980)

67. P.M. Jay, P.G. Coleman, Phys. Rev. A 82, 012701 (2010)

68. R.P. McEachran, A.D. Stauffer, L.E.M. Campbell, J. Phys. B 13, 1281 (1980)

69. B.K. Elza, T.L. Gibson, M.A. Morrison, B.C. Saha, J. Phys. B 22, 113 (1989)

70. R.C. Carvalho, M.T. do N. Varella, M.A.P. Lima, E.P. Silva, J.S.E. Germano, Nucl. Instrum. Methods Phys. Res. Sect. B 171, 33 (2000)

71. W. Tenfen, K.T. Mazon, S.E. Michelin, F. Arretche, Phys. Rev. A 86, 042706 (2012)

72. T. Mukherjee, A.S. Ghosh, A. Jain, Phys. Rev. A 43, 2538 (1991)

73. K.R. Hoffman, M.S. Dababneh, Y.F. Hsieh, W.E. Kauppila, V. Pol, J.H. Smart, T.S. Stein, Phys. Rev. A 25, 1393 (1982)

74. A. Zecca, L. Chiari, E. Trainotti, A. Sarkar, S.dA. Sanchez, M.H.F. Bettega, M.T. do N. Varella, M.A.P. Lima, M.J. Brunger, Phys. Rev. A 85, 012707 (2012)

75. R.W. Wagenaar, A. de Boer, T. van Tubergen, J. Los, F.J. de Heer, J. Phys. B 13, 38551980

76. M.S. Dababneh, Y.F. Hsieh, W.E. Kauppila, C.K. Kwan, S.J. Smith, T.S. Stein, M.N. Uddin, Phys. Rev. A 38, 1207 (1988)

77. K. Floeder, D. Fromme, W. Raith, A. Schwab, G. Sinapius, J. Phys. B 18, 3347 (1985)

78. O. Sueoka, S. Mori, J. Phys. B 19, 4035 (1986)

79. F.A. Gianturco, A. Jain, L.C. Pantano, Phys. Rev. A 36, 4637 (1987)

80. Z. Idziaszek, G.P. Karwasz, Phys. Rev. A 73, 064701 (2006)

81. J.P. Sullivan, C. Makochekanwa, A. Jones, P. Caradonna, S. Buckman, J. Phys. B 41, 081001 (2008)

82. J.O. Hirschfelder, C.F. Curtiss, R.B. Bird, Molecular Theory of Gases and Liquids (Wiley, New York, 1954)

83. E. Clementi, D.L.Raimondi, W.P. Reinhardt, J. Chem. Phys. 38, 2686 (1963)

84. A. Bondi, J. Phys. Chem., 68, 441 (1964)

85. B. Cordero, V. Gómez, A.E. Platero-Prats, M. Revés, J. Echeverría, E. Cremades, F. Barragán, S. Alvarez, in Covalent radii revisited, Dalton Trans. (2008), p. 2832

86. P. Pyykkö, M. Atsumi, Chem. Eur. J. 15, 186 (2009)

87. A. Zecca, L. Chiari, E. Trainotti, M.J. Brunger, J. Phys. B 45, 085203 (2012)

88. D.M. Schrader, Phys. Rev. A 20, 918 (1979)

Open Access This is an open access article distributed under the terms of the Creative Commons Attribution License (http://creativecommons.org/licenses/by/4.0), which permits unrestricted use, distribution, and reproduction in any medium, provided the original work is properly cited. 